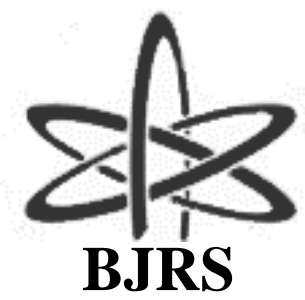

BRAZILIAN JOURNAL

$\mathrm{OF}$

RADIATION SCIENCES

03-01 (2015) 01-13

\title{
Estudo comparativo entre simulações de um sistema de monitoração ocupacional interna utilizando diferentes códigos de Monte Carlo
}

\author{
T. C. F. Fonseca ${ }^{\text {a. }}$ F. M. Bastos ${ }^{\mathrm{a}}$, M. T. T. Figueiredo ${ }^{\mathrm{a}}$, L. S. Souza ${ }^{\mathrm{b}}$, M. C.

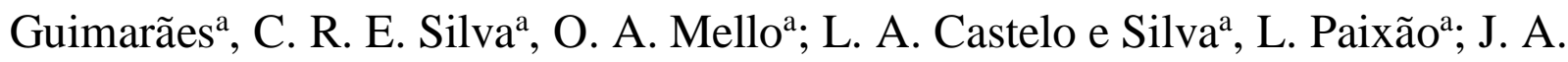 \\ Benavente $^{c}$, F. G. Paiva ${ }^{\mathrm{c}}$ \\ ${ }^{a}$ Programa de Pós-Graduação em Ciências e Tecnologia das Radiações, Materiais e Minerais - CDTN/CNEN \\ ${ }^{b}$ Centro de Desenvolvimento da Tecnologia Nuclear da Comissão Nacional de Energia Nuclear - CDTN/CNEN - \\ Belo Horizonte, MG, Brasil \\ ${ }^{c}$ Curso de Pós-Graduação em Ciências e Técnicas Nucleares da Universidade Federal de Minas Gerais - \\ PCTN/UFMG, Belo Horizonte/MG, Brasil \\ tcff01@gmail.com
}

\begin{abstract}
Computational Monte Carlo (MC) codes have been used for simulation of nuclear installations mainly for internal monitoring of workers, the well known as Whole Body Counters (WBC). The main goal of this project was the modeling and simulation of the counting efficiency (CE) of a WBC system using three different MC codes: MCNPX, EGSnrc and VMC in-vivo. The simulations were performed for three different groups of analysts. The results shown differences between the three codes, as well as in the results obtained by the same code and modeled by different analysts. Moreover, all the results were also compared to the experimental results obtained in laboratory for meaning of validation and final comparison. In conclusion, it was possible to detect the influence on the results when the system is modeled by different analysts using the same MC code and in which MC code the results were best suited, when comparing to the experimental data result.
\end{abstract}

Palavras-chave: MCNPX, EGSnrc, VMC in-vivo, BOMAB, monitoração ocupacional interna. 


\section{INTRODUÇÃO}

Métodos para simulação computacional vêm sendo desenvolvidos e utilizados, em todo o mundo, desde as primeiras décadas do século $\mathrm{XX}^{1}$. Tais métodos compreendem modelos matemáticos e técnicas computacionais que permitem estudar e analisar diversas áreas do conhecimento. Dentre esses métodos, que podem ser contínuos ou discretos, estáticos ou dinâmicos, destaca-se o método de Monte Carlo (MC). MC é um método de simulação estática que utiliza uma sequência de números aleatórios para simular diversos sistemas físicos ${ }^{2}$ e é particularmente interessante na resolução de problemas para os quais não são indicados os métodos determinísticos.

Existem diversos códigos de transporte de partículas que utilizam o método estocástico de Monte Carlo para reproduzir os processos estatísticos de interação atômica e/ou nuclear, gerando aleatoriamente cada uma das muitas partículas primárias e secundárias, a partir de uma fonte radioativa dentro de um sistema. Esses códigos computam todos os eventos, tais como absorção da radiação, espalhamento Compton, radiação de Bremsstrahlung, produção de raios X, entre outros, e reportam os resultados juntamente com seus erros estatísticos. Um desses códigos é o Monte Carlo N-Particle Transport Code (MCNP), que foi criado na década de 1940 pelo Los Alamos National Laboratory, nos EUA ${ }^{3}$. Atualmente, o MCNPX/MCNP6 é uma das últimas versões disponíveis do MCNP e permite o rastreamento de nêutrons, fótons, elétrons, íons leves (deutério, ${ }^{3} \mathrm{He}$ e partículas alfa) e íons pesados ( ${ }^{56} \mathrm{Fe}$ ou ${ }^{238} \mathrm{U}$ ) que "viajam” com diferentes energias cinéticas de até $1 \mathrm{GeV}$. Outro código desenvolvido para fins de simulações computacionais é o Electron-Gamma Shower (EGS) ${ }^{4}$, que simula o transporte de elétrons e fótons de várias energias dentro de uma geometria arbitrária. A primeira versão do EGS foi lançada em meados de 1974. A versão atual, cujo nome foi modificado para EGSnrc: software tool to model radiation transport", é mantida pela National Research Council Canada ${ }^{5}$. Um terceiro código, o Visual Monte Carlo (VMC) in-vivo, foi desenvolvido por J. Hunt, et al. do Instituto de Radioproteção e Dosimetria (IRD/RJ) e é utilizado para simular sistemas de monitoração, conhecidos por Contadores de Corpo Inteiro (CCI), para fins de calibração e, bem como, para cálculo de dose para diferentes configurações de fontes de radiação ${ }^{6}$.

A utilização de técnicas in vivo em dosimetria interna consiste na determinação qualitativa e quantitativa de substâncias radioativas depositadas internamente no corpo humano ou em órgãos específicos, quando há risco iminente de contaminação dos trabalhadores de instalações nucleares. Este procedimento é executado nos CCI, que são formados basicamente por um detector e um sistema associado que permite medir a energia da radiação emitida dos radionuclídeos que as produziram. A calibração dos CCI é 
realizada utilizando simuladores físicos como, por exemplo, o $\mathrm{BOMAB}^{7}$ para determinar o fator de eficiência de contagem do sistema, ou por meio de sistemas computacionais que permitem a criação dos modelos matemáticos, que correspondem a geometria dos simuladores físicos e da fonte de radiação $0^{8,9}$. O objetivo desse trabalho foi modelar e simular um sistema de Contador de Corpo Inteiro (CCI), e calcular a eficiência de contagem, utilizando os códigos MCNPX, EGSnrc e VMC in-vivo. Além disso, dados experimentais obtidos no Laboratório de Dosimetria Interna do CDTN/CNEN foram utilizados para validação e comparação final dos resultados obtidos por simulação. 


\section{MATERIAIS E MÉTODOS}

Neste estudo, o sistema físico de CCI, apresentado na Figura 1, foi o modelo das simulações e é constituído por um simulador BOMAB e um detector $\mathrm{NaI}(\mathrm{Tl})$ 8” $\mathrm{x} 4$ ” posicionado a uma altura fixa em relação a cama, de 0,532 m. Medidas foram realizadas alterando a posição do detector longitudinalmente em relação ao BOMAB. Foram escolhidas 8 posições em intervalos de 0,20 m, a partir da primeira posição ajustada de tal forma que o centro da base do detector ficasse a $0,1 \mathrm{~m}$ da borda da cabeça do simulador.

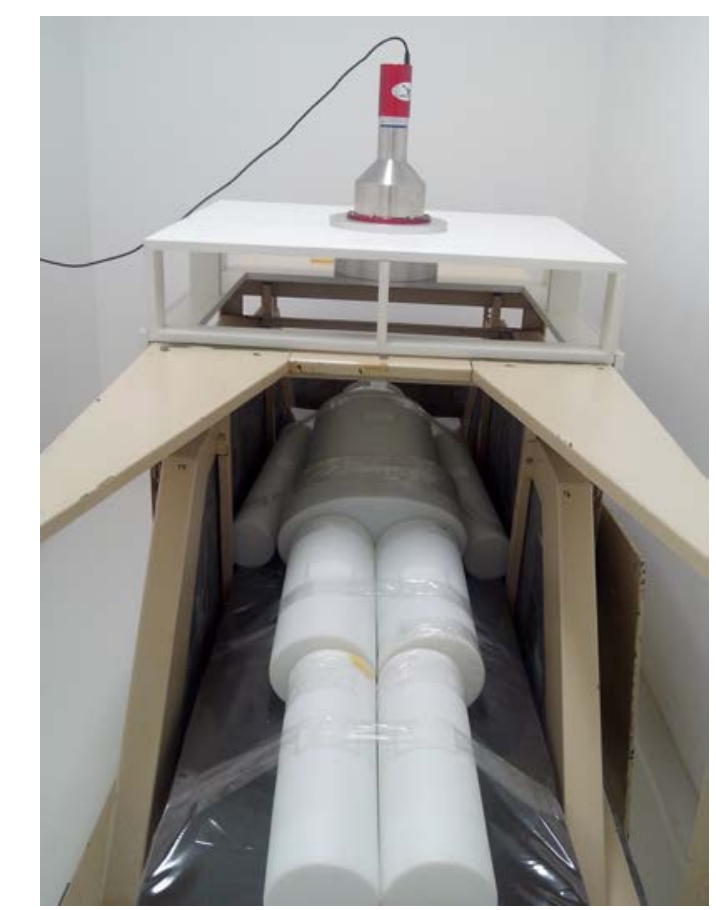

Figura 1: Sistema CCI composto por uma cama, o simulador BOMAB e um detector.

O detector cintilador $\mathrm{NaI}(\mathrm{Tl})$ de dimensões 8”x4” tem forma cilíndrica, com altura de 0,1016 m e diâmetro da base de 0,2032 $\mathrm{m}$ e é revestido por uma camada de alumínio de $1,5 \times 10^{-3} \mathrm{~m}$ de espessura. O simulador antropomórfico BOMAB masculino é formado por cilindros elípticos e esféricos de poliamida [ $\mathrm{NH}\left(\mathrm{CH}_{2}\right)_{5} \mathrm{CO}$ com densidade de $1,15 \times 10^{3} \mathrm{~kg} \cdot \mathrm{m}^{-3}$ e espessura de $5,0 \times 10^{-3} \mathrm{~m}$. O BOMAB tem aproximadamente $1,70 \mathrm{~m}$ de altura e $70 \mathrm{~kg}$ de massa. A Tabela 1 
apresenta as dimensões das partes que o compõem ${ }^{10}$. Para este estudo, suas cavidades foram preenchidas por uma solução de $\mathrm{KCl}$, para verificação da presença do radioisótopo potássio-40.

Tabela 1: Dimensões do objeto simulador BOMAB.

\begin{tabular}{lllll}
\hline Parte & 2a $\mathbf{( m )}$ & $\mathbf{2 b}(\mathbf{m})$ & $\mathbf{h ~ ( m )}$ & Volume $(\mathbf{L})$ \\
\hline Cabeça & 0,191 & 0,146 & 0,197 & 4,301 \\
Pescoço & 0,148 & 0,137 & 0,095 & 1,511 \\
Tórax & 0,298 & 0,207 & 0,42 & 20,133 \\
Pelve & 0,362 & 0,197 & 0,202 & 11,282 \\
Braço & 0,095 & 0,095 & 0,581 & 4,136 \\
Coxa & 0,162 & 0,149 & 0,400 & 7,537 \\
Perna & 0,126 & 0,121 & 0,398 & 4,762 \\
\hline
\end{tabular}

Paralelamente à parte experimental, o sistema físico de CCI foi modelado e simulado por três grupos de pesquisadores, denominados $G_{1}, G_{2}$ e $G_{3}$, utilizando três códigos computacionais MC diferentes: MCNPX, EGSnrc e VMC in-vivo. Os grupos $\mathrm{G}_{1}$ e $\mathrm{G}_{2}$ utilizaram os códigos MCNPX e VMC in-vivo e o grupo $\mathrm{G}_{3}$ utilizou os códigos MCNPX e EGSnrc.

Os grupos modelaram todo o sistema CCI: BOMAB e detector, bem como o reposionamento do detector nas 8 distintas posições medidas no processo experimental. No processo de modelagem utilizando os códigos MCNPX e EGSnrc, a geometria do CCI foi desenvolvida de maneira distintas entre os grupos. Os grupos $G_{1}$ e $G_{3}$ modelaram o BOMAB com a sua superfície tangente ao plano de apoio, conforme realizado experimentalmente e mostrado nas Figuras 2a e 2b. O grupo $\mathrm{G}_{2}$ modelou o BOMAB centrando a cabeça, pescoço, tórax e pelves em um único eixo, distribuindo uniformemente os braços, coxas e pernas, dos lados direito e esquerdo do simulador conforme mostrado na Figura 2c. A Figura 3 mostra as posições do detector para as quais foram realizadas as simulações, conforme o procedimento experimental. 


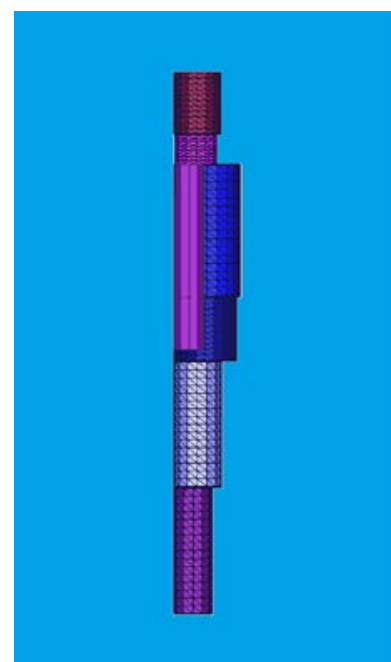

$\mathrm{a}$

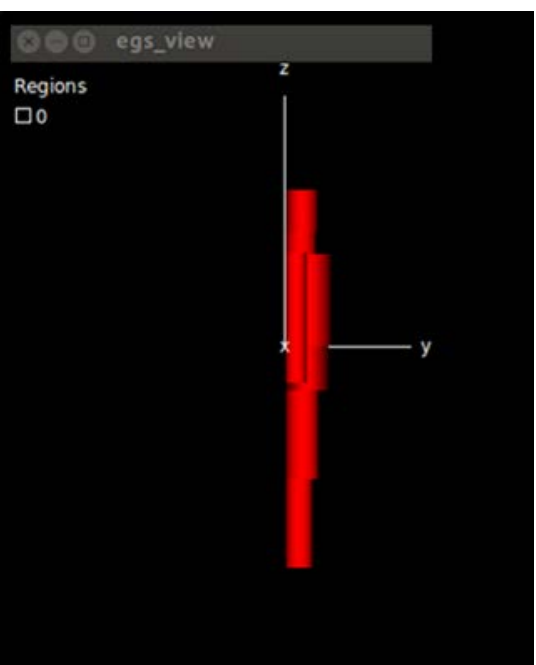

b

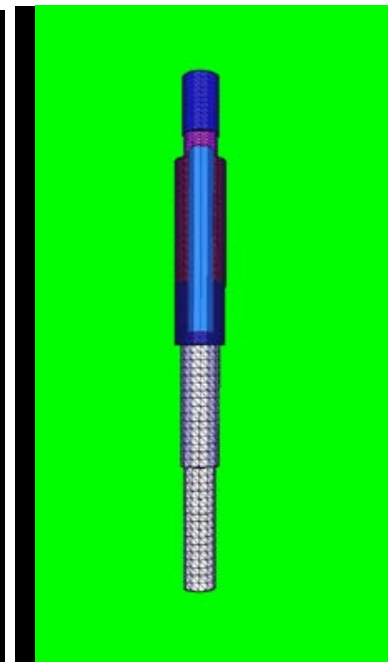

$\mathrm{C}$

Figura 2: Modelagem do BOMAB nas metodologias adotadas pelos $G_{1}$ e $G_{3}$ : (a) MCNPX e (b) EGSnrs; e pelo $G_{2}$ : (c) MCNPX.

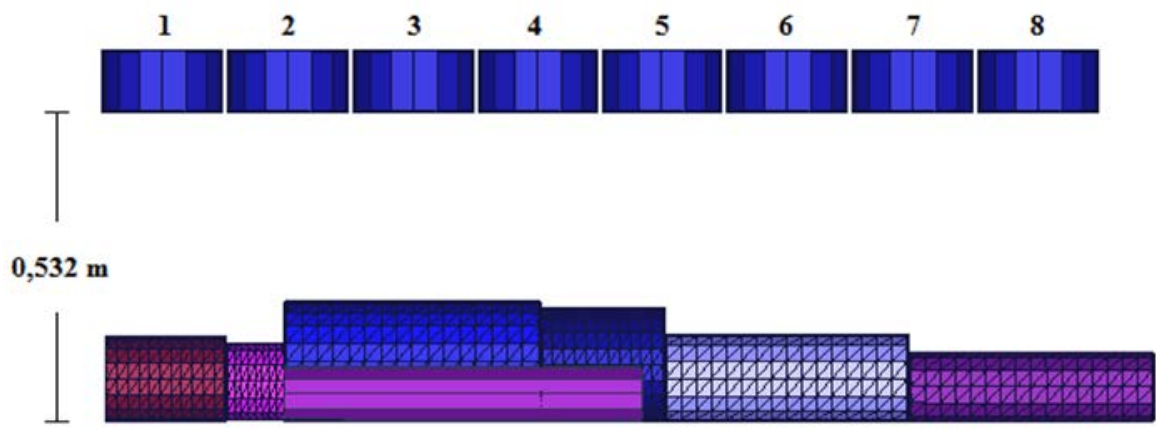

Figura 3: Modelagem do simulador BOMAB apresentado todas as oito posições do detector 8 " $x$ "”.

A composição elementar e a densidade dos materiais utilizados nas simulações com os códigos MCNPX e EGSnrc são apresentadas na Tabela 2. Por outro lado, o VMC in-vivo possui sua própria biblioteca de materiais e densidades que usualmente são empregados nos procedimentos de calibração para CCI. 
Tabela 2: Composição elementar dos materiais utilizados nas simulações com os códigos MCNPX e EGSnrc.

\begin{tabular}{ccccc}
\hline Elemento & $\begin{array}{c}\text { Ar } \\
\mathbf{( \% )}\end{array}$ & $\begin{array}{c}\text { Plástico (BOMAB) } \\
\mathbf{( \% )}\end{array}$ & $\begin{array}{c}\text { Cristal } \\
\mathbf{( \% )}\end{array}$ & $\begin{array}{c}\text { Água } \\
\mathbf{( \% )}\end{array}$ \\
\hline $\mathrm{H}$ & - & 0,0980 & - & 0,1119 \\
$\mathrm{C}$ & 0,0001 & 0,6369 & - & - \\
$\mathrm{N}$ & 0,7553 & 0,1238 & - & - \\
$\mathrm{O}$ & 0,2318 & 0,1414 & - & 0,8881 \\
$\mathrm{Na}$ & - & - & 0,4850 & - \\
$\mathrm{Ar}$ & 0,0128 & - & - & - \\
$\mathrm{I}$ & - & - & 0,4850 & - \\
$\mathrm{Tl}$ & - & - & 0,0300 & - \\
\hline$\rho\left(\mathrm{kg} \cdot \mathrm{m}^{-3}\right)$ & 1,205 & $1,15 \times 10^{3}$ & $3,67 \times 10^{3}$ & $1,0 \times 10^{3}$ \\
\hline
\end{tabular}

Uma vez que no procedimento experimental foi utilizada uma solução de $\mathrm{KCl}$, as simulações focaram o estudo da interação de fótons com energia de 1,46 MeV, devido a presença de potássio-40 na solução. As simulações consideraram uma fonte volumétrica uniformemente distribuída nas cavidades do simulador BOMAB. A Figura 4 mostra uma representação gráfica da interação dos fótons, utilizando o software Vised ${ }^{11}$.

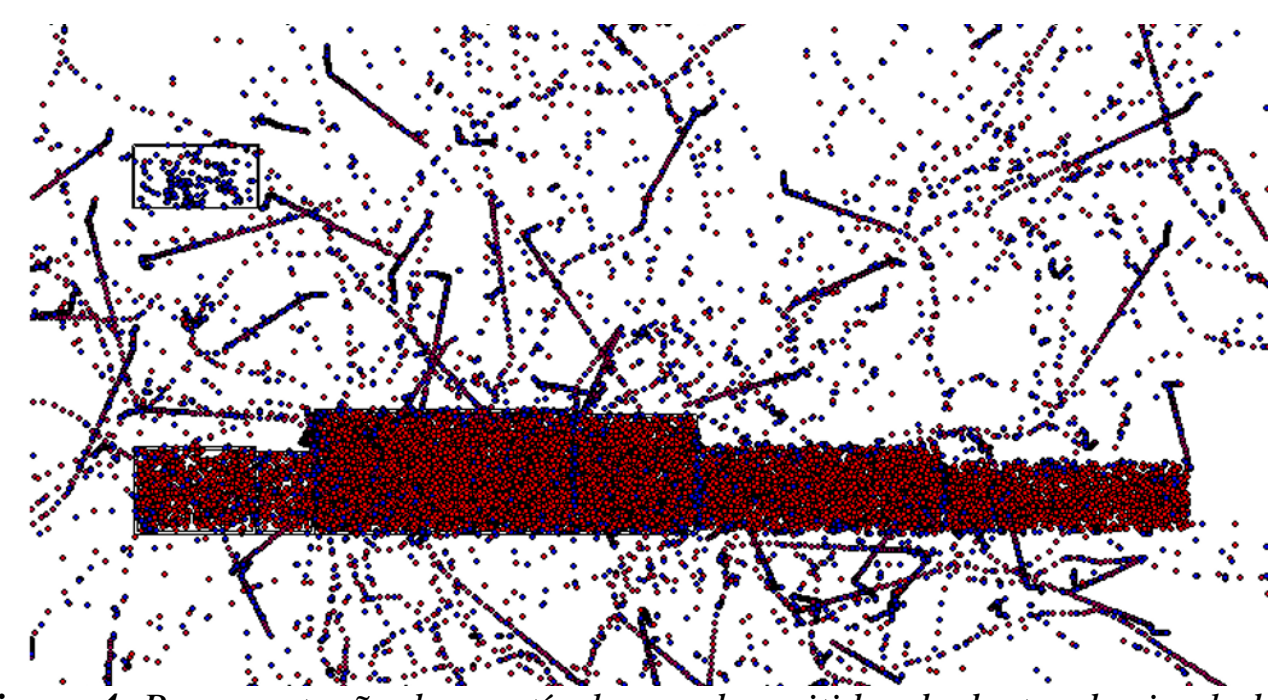

Figura 4: Representação das partículas sendo emitidas de dentro do simulador BOMAB e as interações de fótons com o detector $\mathrm{NaI}(\mathrm{Tl})$ na posição um. 
Os códigos MCNPX, VMC in-vivo e EGSnrc calculam uma distribuição de altura de pulso (DAP) a partir da interação dos fótons no volume sensível do detector $\mathrm{NaI}(\mathrm{Tl})$. O código MCNPX determinou a DAP utilizando a tally F8. Para obter uma DAP no EGSnrc, foi derivada uma aplicação da classe EGS Advanced Application, a partir da aplicação tutor7pp, que possui uma função implementada para obtenção de tais distribuições. A eficiência de contagem do

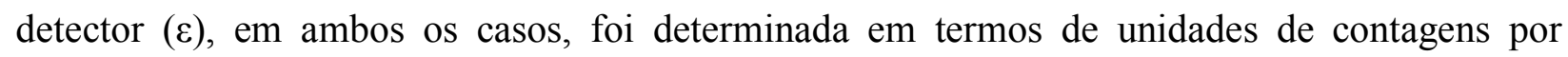
números de partículas emitidas (contagens/nps). O software VMC in-vivo calcula o fator de calibração em unidades de becquerel por contagens por segundos $(\mathrm{Bq} / \mathrm{cps})$, que pode ser facilmente convertido em eficiência de contagem se considerarmos que o número de partículas emitidas pela fonte (nps) é igual a Becquerel (Bq). Para minimizar os erros relativos calculados pelos códigos o número de histórias ou partículas emitidas pela fonte em todas as simulações foi de $10^{8} 12$.

\section{RESULTADOS E DISCUSSÕES}

A Tabela 3 e a Figura 5 apresentam os valores obtidos para a eficiência de contagem nas simulações com os códigos MCNPX, EGSnrc e VMC in-vivo e nos experimentos no CCI do Laboratório de Dosimetria Interna do CDTN, para as oito posições do detector.

Tabela 3: Tabela 3. Resultados em termos de eficiência de contagem obtidos com os códigos MCNPX, VMC in-vivo e EGSnrc.

\begin{tabular}{|c|c|c|c|c|c|c|c|}
\hline \multicolumn{8}{|c|}{$\varepsilon\left(x 10^{-3}\right.$ contagem por $\gamma$ emitida $)$} \\
\hline \multirow{2}{*}{ Posição } & \multicolumn{2}{|c|}{ VMC } & \multicolumn{3}{|c|}{ MCNPX } & \multirow{2}{*}{$\begin{array}{c}\text { EGS } \\
\mathbf{G}_{3}\end{array}$} & \multirow[t]{2}{*}{ Experimental } \\
\hline & $\mathrm{G}_{1}$ & $\mathbf{G}_{2}$ & $\mathbf{G}_{1}$ & $\mathbf{G}_{2}$ & $\mathbf{G}_{3}$ & & \\
\hline 1 & 1,19 & 1,20 & 1,01 & 1,09 & 1,09 & 1,01 & 0,96 \\
\hline 2 & 1,69 & 1,76 & 1,46 & 1,52 & 1,59 & 1,42 & 1,37 \\
\hline 3 & 2,09 & 2,22 & 1,80 & 1,91 & 1,95 & 8,39 & 1,73 \\
\hline 4 & 2,23 & 2,37 & 1,92 & 2,02 & 2,07 & 1,07 & 1,91 \\
\hline 5 & 2,12 & 2,27 & 1,83 & 1,93 & 1,98 & 1,27 & 1,90 \\
\hline 6 & 1,79 & 1,92 & 1,59 & 1,68 & 1,70 & 1,22 & 1,66 \\
\hline 7 & 1,48 & 1,57 & 1,31 & 1,42 & 1,44 & 1,06 & 1,41 \\
\hline
\end{tabular}




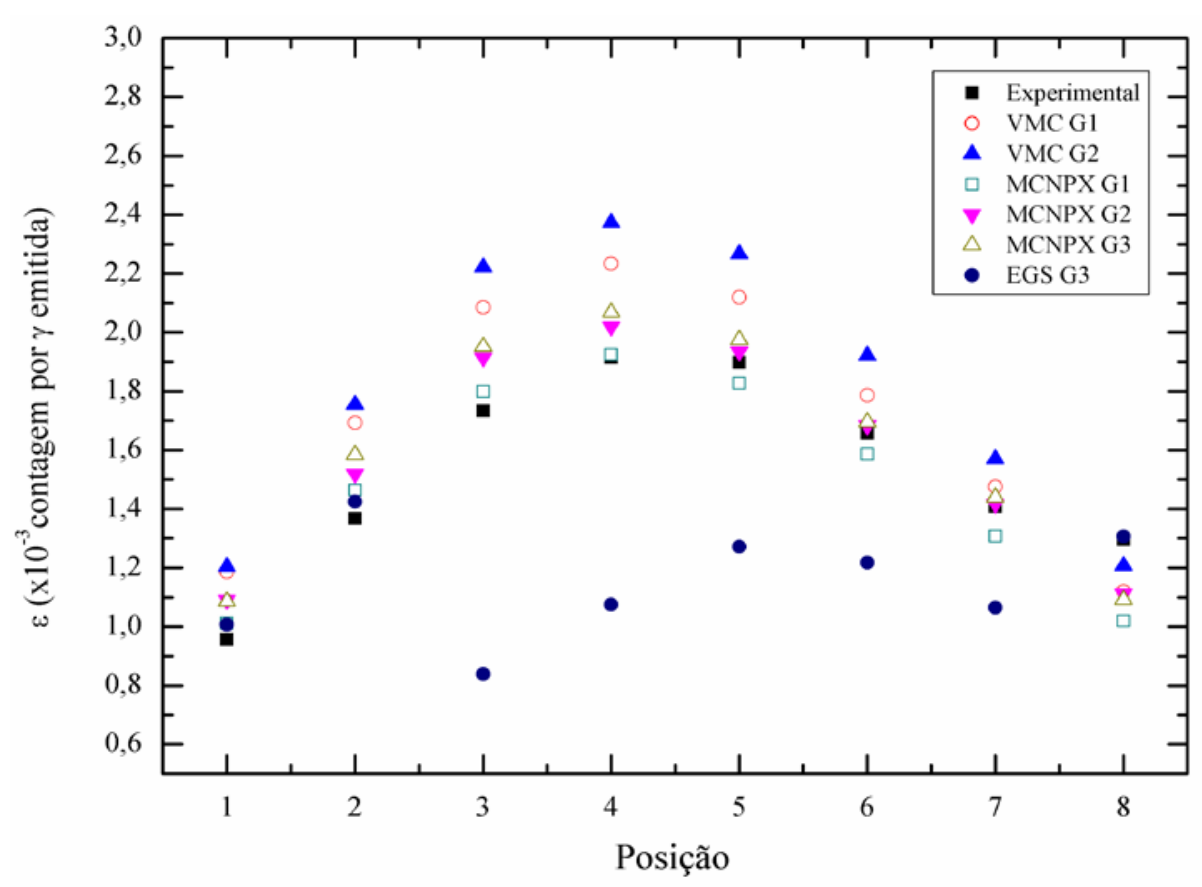

Figura 5: Resultados em termos de eficiência de contagem obtidos com os códigos MCNPX, VMC in-vivo e EGSnrc.

A Tabela 4 apresenta as diferenças percentuais entre os valores de eficiência de contagem obtidos entre grupos diferentes para o mesmo código (MCNPX e VMC in-vivo) e as diferenças percentuais entre códigos diferentes, para um mesmo grupo.

Tabela 4: Diferenças percentuais entre os valores de eficiência de contagem obtidos com os códigos MCNPX, VMC e EGSnrc.

\begin{tabular}{|c|c|c|c|c|c|c|c|}
\hline \multicolumn{5}{|c|}{$\begin{array}{c}\text { Comparação entre grupos } \\
(\%)\end{array}$} & \multicolumn{3}{|c|}{$\begin{array}{c}\text { Comparação entre códigos } \\
(\%)\end{array}$} \\
\hline \multicolumn{5}{|c|}{$\left.\left(100 \times\left(G_{i}-G_{j}\right) / G_{j}\right) \%\right)$} & \multicolumn{3}{|c|}{$\left.\left(100 \times\left(\operatorname{Cod}_{i}-\operatorname{Cod}_{j}\right) / \operatorname{Cod}_{j}\right) \%\right)$} \\
\hline & VMC & MCNPX & MCNPX & MCNPX & G1 & G2 & G3 \\
\hline Posição & $\mathbf{G}_{1} \mathbf{e} \mathbf{G}_{2}$ & $\mathbf{G}_{1} \mathbf{e} \mathbf{G}_{2}$ & $\mathbf{G}_{1} \mathbf{e} \mathbf{G}_{3}$ & $\mathbf{G}_{2} \mathbf{e} \mathbf{G}_{3}$ & $\begin{array}{c}\text { VMC e } \\
\text { MCNPX }\end{array}$ & $\begin{array}{l}\text { VMC e } \\
\text { MCNPX }\end{array}$ & $\begin{array}{c}\text { MCNPX e } \\
\text { EGSnrc }\end{array}$ \\
\hline 1 & 1 & 7 & 7 & 0 & 18 & 10 & 8 \\
\hline 2 & 4 & 4 & 8 & 4 & 16 & 16 & 12 \\
\hline 3 & 6 & 6 & 8 & 2 & 16 & 16 & 77 \\
\hline 4 & 6 & 5 & 7 & 2 & 16 & 17 & 94 \\
\hline
\end{tabular}




\begin{tabular}{cccccccc}
$\mathbf{5}$ & 7 & 5 & 8 & 3 & 16 & 18 & 56 \\
$\mathbf{6}$ & 7 & 5 & 7 & 1 & 13 & 14 & 39 \\
$\mathbf{7}$ & 6 & 8 & 9 & 1 & 13 & 11 & 36 \\
$\mathbf{8}$ & 7 & 8 & 6 & 2 & 10 & 9 & 17 \\
\hline
\end{tabular}

As diferenças percentuais, entre os valores de eficiência de contagem $(\varepsilon)$, foram calculadas de tal forma que se obtivesse a maior diferença percentual entre os valores comparados.

Na comparação dos resultados obtidos entre os grupos $\mathrm{G}_{1}$ e $\mathrm{G}_{2}$ e o código VMC in-vivo, observou-se uma diferença máxima de 7\%. Comparando os resultados obtidos com o MCNPX a diferença máxima foi de $8 \%$ entre $\mathrm{G}_{1}$ e $\mathrm{G}_{2}, 9 \%$ entre $\mathrm{G}_{1}$ e $\mathrm{G}_{3}$ e $4 \%$ entre $\mathrm{G}_{2}$ e $\mathrm{G}_{3}$. Observa-se que as diferenças encontradas podem ser associadas aos fatores humanos, ou seja quando o mesmo sistema é modelado por diferentes analistas, somado a flutuações estatísticas de cada simulação. Por outro lado, quando os resultados são comparados entre códigos distintos, VMC in-vivo e MCNPX, a diferença percentual máxima foi de $18 \%$ tanto para $G_{1}$ quanto para $G_{2}$. Comparando os códigos MCNPX e EGSnrc a diferença máxima obtida foi de 94\%. Observou-se que as diferenças percentuais nas posições do tórax e pelve, ou seja, partes do simulador com maiores volumes, apresentaram uma discrepância maior quando comparadas com os resultados experimentais e os outros códigos. Uma possível explicação para esta diferença poderia estar associada à descrição da fonte no arquivo de input e, por consequência, na emissão dos fótons gerados pelo código EGSnrc. No entanto, não houve um suporte adequado dos responsáveis pelo código para confirmar esta suposição. É possível que o EGSnrc não seja um código apropriado para este tipo de simulação.

Na comparação dos resultados experimentais com o código MCNPX foram obtidas diferenças nas faixas de 1 a 22\%, 1 a 15\% e 2 a $16 \%$, para os grupos $G_{1}, G_{2}$ e $G_{3}$, respectivamente. Por outro lado, o código VMC in-vivo apresentou diferenças nas faixas de 5 a 24\% e 7 a 29\% para os grupos $G_{1}$ e $G_{2}$, respectivamente. E finalmente, o EGSnrc apresentou diferenças na faixa de 1 a $44 \%$, e uma diferença de 385\% para a posição 3. Cabe ressaltar, que estes valores, com exceção dos resultados obtidos para as posições 3, 4 e 5 com o código EGSnrc, encontram-se dentro da faixa de aceitação $( \pm 25 \%)$, conforme o relatório escrito pela ANSI N 13.30, para calibração de sistema como o $\mathrm{CCI}^{10}$. 


\section{CONCLUSÕES}

Os resultados obtidos para grupos distintos com o mesmo código, tanto no MCNPX quanto no VMC in-vivo apresentaram diferenças percentuais relevantes. Essas diferenças podem ser atribuídas as diferentes concepções da geometria do sistema de CCI e ao entendimento do problema, que depende do programador.

Os resultados obtidos com os códigos computacionais MCNPX, VMC in-vivo e EGSnrc em relação aos calculados experimentalmente encontram-se na faixa de aceitação, com exceção dos obtidos com o código EGSnrc nas posições 3, 4 e 5. A falta de suporte dos desenvolvedores do código não permitiu uma descrição mais adequada da fonte e da emissão dos fótons nas diferentes partes do BOMAB e, por isso, observou-se um aumento nas diferenças percentuais nas posições do detector que sofrem maior influência do tórax e pelve.

\section{AGRADECIMENTO}

Os autores agradecem à CNEN, CAPES, FAPEMIG pelas bolsas de mestrado e doutorado e ao Instituto de Radioproteção e Dosimetria (IRD) pelo suporte com o simulador BOMAB. Pesquisa desenvolvida com o apoio do LN/IRD. 


\section{REFERÊNCIAS}

1. PIDD, M. Computer Simulation in Management Science. $4^{\text {th }}$ ed. England: John Wiley \& Sons; 2003.

2. METROPOLIS, N; ULAM, S. The Monte Carlo Method. J Amer Stat Assoc, 1949, 44: 335-41.

3. BRIESMEITER, J. F. et. al. MCNP-4B - A General Monte Carlo N-Particle Transport Code-Version 4B. Los Alamos National Laboratory, LA-12625-M. 1997.

4. NELSON, W. R.; HIRAYAMA, H.; ROGERS, D. W. The EGS4 Code System. Report SLAC-265, Stanford Linear Accelerator Center. Stanford, CA. 1985.

5. KAWRAKOW, I.; MAINEGRA-HING, E.; TESSIER, F.; WALTERS, B. R. B. The EGSnrc C ${ }^{++}$class library. NRC Report PIRS-898 (rev A). NRCC. 2009.

6. HUNT, J. G.; DANTAS, B. M.; AZEREDO, A. M. Visual Monte Carlo in-vivo in the CONRAD and IAEA Whole Body Counter Intercomparisons. In. WORK ON UNCERTAINTY ASSESMENT IN COMPUTATIONAL DOSIMETRY, 2007, Bologna.

7. BUSH, F. The integral dose received from a uniformly distributed radioactive isotope. British J Radiol, v. 22, P. 96-102, 1949.

8. FONSECA, T. C. F.; BOGAERTS, R.; HUNT, J.; VANHAVERE, F. A. Methodology to develop computational phantoms with adjustable posture for WBC calibration. Phys. Med. Biol. v. 59(22), p. 6811-6825, 2014.

9. ICRU - International Commission on Radiation Units and Measurements. Phantoms and computational models in therapy, diagnosis and protection. ICRU Report 48, Bethesda: ICRU, 1992. 194 p. 
10. ANSI - American National Standards Institute, Performance Criteria for Radiobioassay, ANSI N 13.30, New York. 1996.

11. VISED X_22S: Visual Editor for interactively constructing \& visualizing MCNPX geometry. Disponível em: <http://www.mcnpvised.com/> Último acesso: 20 Jan. 2015.

12. X-5 Monte Carlo Team. LA-UR-03-1987: MCNP - A General Monte Carlo N-Particle Transport Code, version 5, volume I: Overview and Theory. 2003 (Revised 2/1/2008). 